\title{
The Multiplier Effect of Applied Machine Learning Technology in Modern Healthcare
}

\author{
Abhijit Bandyopadhyay \\ Teacher-in-Charge, Raniganj Institute of Computer and Information Science (RICIS), Raniganj, West Bangal, India
}

Corresponding author: abhijit.ricis@gmail.com

Received: $28-03-2020$

Revised: 24-04-2020

Accepted: 05-06-2020

\begin{abstract}
In this concept paper on machine learning and its application in modern healthcare, an attempt has been made to analyze the uses of machine learning in various health information technology and e-health paradigms and the multiple benefits accrued from applied machine learning in the diversified domain of modern healthcare. In juxtaposition with Artificial Intelligence, Data Mining, Optimization, and Statistics, machine learning paradigms have the potential to meaningfully revolutionize the modern healthcare system. Uses of artificial intelligence, big data analytics, data mining, statistical optimization techniques, and, modelling have the potential to improve modern healthcare to new levels of efficient and effective management of healthcare. Healthcare is a prime mover in any nation's progress and application of machine learning technology accentuates the growth and efficacy of this crucial sector. Connecting global resources of healthcare professionals and cutting-edge healthcare technology through the seamless integration of skilled manpower, infrastructural facilities, investment options and, the world pool trained medicos may usher in a new era of MLT-enabled healthcare system that reduces the cost, optimizes the processes and delivers the best across boundaries. Hence machine learning adds multiple benefits to the modern healthcare system, as referred to as its "multiplier effect". In this concept paper, a sincere effort has been made to relate the basics of MLT, how it can be integrated with health information technology and, the manners in which it can transform people's lives with the timely and proper intervention. It has been proved in this concept paper that MLT has a multiplier effect on the judicious delivery of quality and affordable healthcare. Although it has also been delineated that the best tool in HIT is still the brain of medical practitioners, it cannot also be denied that the adoption pf MLT and thoughtful extrapolation with the medical database can improve our healthcare system manifold. Hence a trustworthy application of MLT tools can help medical professionals taking better decisions in a timely, cost-effective and, reliable manner. The journey from reactive theoretical antidotes to predictive analytical solutions in healthcare is the precious boon derived from advances in machine learning technology. The medical database and EMRs can pave the road for stunningly accurate and optimized healthcare solutions provided MLT is synchronized with HIT and properly applied by adequately trained professionals. Hopefully, it can also be expected that our healthcare
\end{abstract}


revolution is just a stone's throw away if MLT and modern HIT are juxtaposed most professionally. This multiplicity of benefits MLT provides to HIT culminates into the proverbial "multiplier effect".

Keywords: Machine Learning, Artificial Intelligence, Data Mining, Optimization, Training Data, Algorithm, Connectionism, Artificial Neural Networks, Bioinformatics, Prophylactic, Therapeutic, DNA Sequence, Structural Health Monitoring

Coined by Arthur Samuel, a pioneer in computer gaming and artificial intelligence, in 1959, machine learning came a long way through pattern recognition and the development of neural networks that can recognize 40 characters including 26 alphabets, 10 digits and, 4 special symbols from a computer terminal. Over the years it developed into a full-fledged discipline now. Machine learning is the study of computer algorithms that use artificial intelligence and build mathematical models based on training data [1]. It is used to predict and take decisions in email filtering and computer vision where the development of conventional algorithms is difficult or infeasible. Using computational statistics and mathematical optimization, data mining is done is exploratory data analysis through unsupervised learning is carried out to deliver predictive analysis. It is the self-learning method by computers, hence called machine learning. Through machine learning, computers can discover to perform tasks without being explicitly programmed [4]. Algorithms are designed whereby computers can execute problem-solving in a step by step method without actually developing a specific program set as human programmers need to do. The Machine learning discipline employs various approaches to accomplish tasks like digital character recognition even though a fully satisfactory algorithm is neither available nor feasible to develop one [7].

Machine learning has grown out of the constant upgrading of the flowing. These can be described keeping in mind the famous quote of Alan Turing.

"Can machines think?"

---- Alan Turing (Computing Machinery and Intelligence)

\section{Artificial Intelligence}

Researchers of artificial intelligence wanted machines to learn by themselves and they applied perceptron and neural networks as an extension of generalized linear models of statistics [5]. Probabilistic reasoning was particularly employed in medical diagnosis. But practical problems of data acquisition and representation called for a logical knowledge-based approach [32]. So, statistical models were replaced by expert systems employing artificial intelligence. Inductive logic programming came into use for pattern recognition and information retrieval. Neural networks were abandoned and back propagation was introduced by some phenomenal AI researchers like Hopfield, Rumelhart, and, Hinton [8]. By adopting connectionism and abandoning symbolic approaches, artificial embraced probability theory, and gave rise to augmented machine learning to solve problems of practical nature as a separate and distinguished field of immense importance.

\section{Data Mining}

Machine learning owes many contributions to data mining [2]. Whereas they overlap significantly, the clear distinction is felt when data mining discovers new insights about data set but machine learning 
focuses on prediction over the training data. Unsupervised learning by machine learning is accentuated by the reproduction of known data amply supported by the discovery of new data through artificial intelligence. Hence the two fields interact and enrich one another [11].

\section{Optimization}

Optimization and machine learning go hand in hand in solving practical problems in a big way. While optimization minimizes the loss on a training set machine learning minimizes loss on unseen samples. The minimization of loss function tries to remove the discrepancy between prediction and actual results [9].

\section{Statistics}

Statistics help to draw inferences based on sample data or training data while machine learning tries to figure out generalizable predictive patterns [3]. As a result, a new field from data science has emerged called statistical earning that amalgamates these two fields. Between the two statistical modelling paradigms, the data model remained in the purview of inferential statistics whereas an algorithmic model led to machine learning [12].

\section{APPROACHES OF MACHINE LEARNING}

As Tom M Mitchell puts it:

"A computer program is said to learn from experience $\mathrm{E}$ with respect to some class of tasks $\mathrm{T}$ and performance measure $\mathrm{P}$ if its performance at tasks in T, as measured by $\mathrm{P}$, improves with experience E". Machine learning depends on the nature or feedback of the learning system [Fig. 1]. Based on this, machine learning has three categories of approaches:

\section{Supervised learning}

* Unsupervised learning

Reinforcement learning

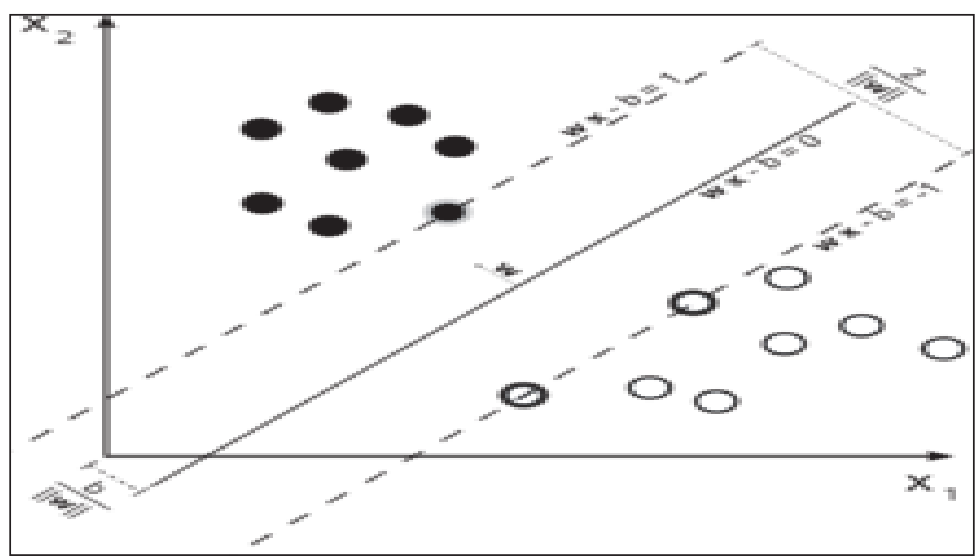

Fig. 1: Machine Learning Algorithms 
In supervised learning, the computers are given example inputs and desired outputs by the 'teacher' so that inputs and outputs are mapped and computers learn a general rule. In unsupervised learning, no label is given for learning, algorithm and computers learn on its own to find the input structure and discover the hidden patterns of the data and even develop 'feature learning' [31]. In reinforcement learning, a computer interacts dynamically with its environment and a certain goal is performed like driving a vehicle (Self-driving vehicles) or playing video games by navigating problem space and maximizing rewards by an analogous feedback mechanism. Some other approaches that combine the above three approaches and are used often include topic modelling, dimensionality reduction, and, meta-learning. Most recently, in 2020 deep learning has been introduced as a machine learning approach having far-reaching positive consequences.

\section{(A) Machine Learning Algorithms and Models}

Different types of machine learning algorithms are:

\section{Supervised learning}

In the supervised learning model, data is separated into regions by a linear boundary. In the following figure. [Fig. 2], the linear boundary divides black circles from the white. Both the inputs and desired output are contained in a mathematical model built by supervised learning. This data is called training data that gives the supervisory signal. Training data is represented by a matrix while an array or vector called feature vector represents the training example [34]. A Supervised learning algorithm learns an objective function by iterative optimization. The algorithm is said to perform the task when it improves the accuracy of outputs called predictions. Different types of supervised learning include (a) classification, that provides a limited set of values and, (c) regression that provides any numerical value.

\section{Supervised Learning as Function Approximation}

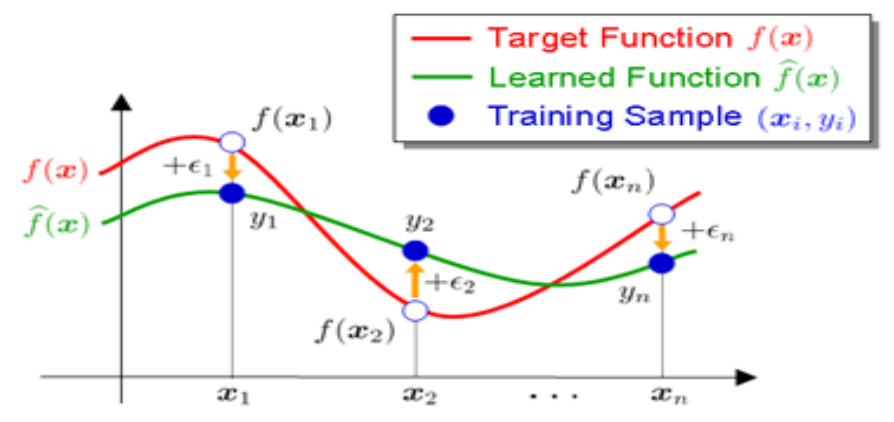

Goal: Learn $f(\boldsymbol{x})$ from $\left\{\left(\boldsymbol{x}_{i}, y_{i}\right)\right\}_{i=1}^{n}$

Fig. 2: Supervised Learning 


\section{Unsupervised learning}

Containing only inputs this type of machine learning learns from test data based on commonalities and reacts accordingly [Fig. 3]. In density application in statistics and probability density functions, it summarizes and explains data features as done in cluster analysis. Between supervised and unsupervised there is also semi-supervised learning that works on missing training labels yet provides considerable improvement in learning accuracy.

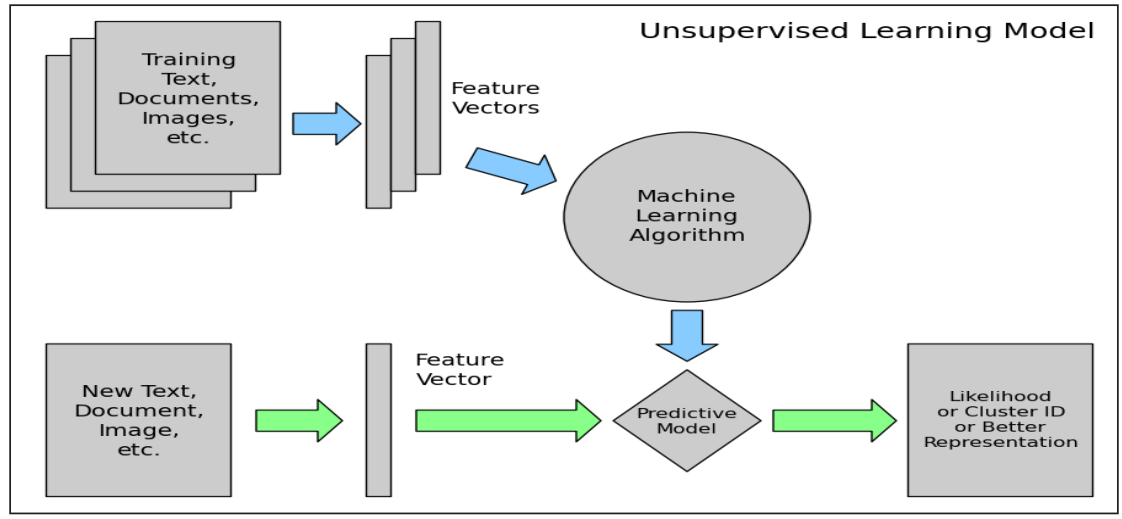

Fig. 3: Unsupervised Learning

\section{Reinforcement learning}

This type of machine learning is concerned about software agents taking action striving for cumulative rewards. It finds application in game theory, control theory, operations research, simulation-based optimization, SWAM intelligence and, genetic algorithms [Fig. 4]. In the Markov Decision Process (MDP) environment, dynamic programming techniques are used and its applications include autonomous vehicles like driverless cars.

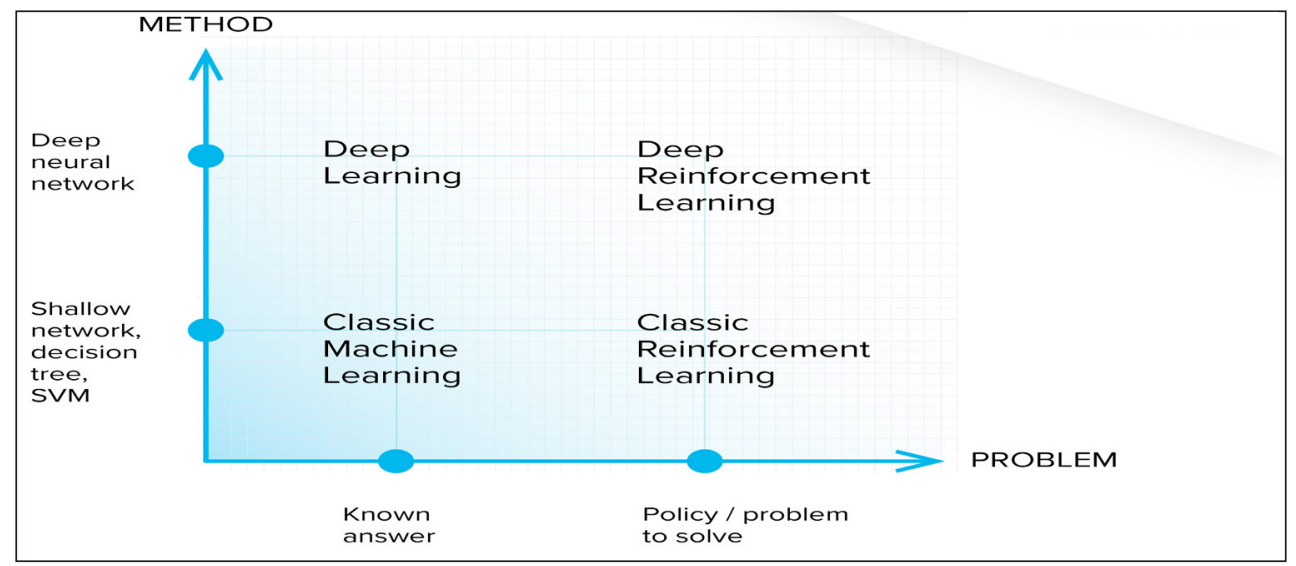

Fig. 4: Reinforcement Learning 


\section{(B) Models of Machine Learning}

The models are an integral part of machine learning but they require a lot of data to perform meaningfully [10]. To come at a predictive part, machine learning involves the artificial neural network as the guiding force behind a predictive analysis. Just like neurons or nerve cells in the human brain, an artificial neural network is a vast network of an interconnected group of nodes that represent artificial neurons [16]. One artificial neuron's output connected to the input of another is represented by an arrow as shown in the figure below [Fig. 5]. Some of the applications of Artificial Neural Network are computer vision, speech recognition, and, medical diagnosis [14].

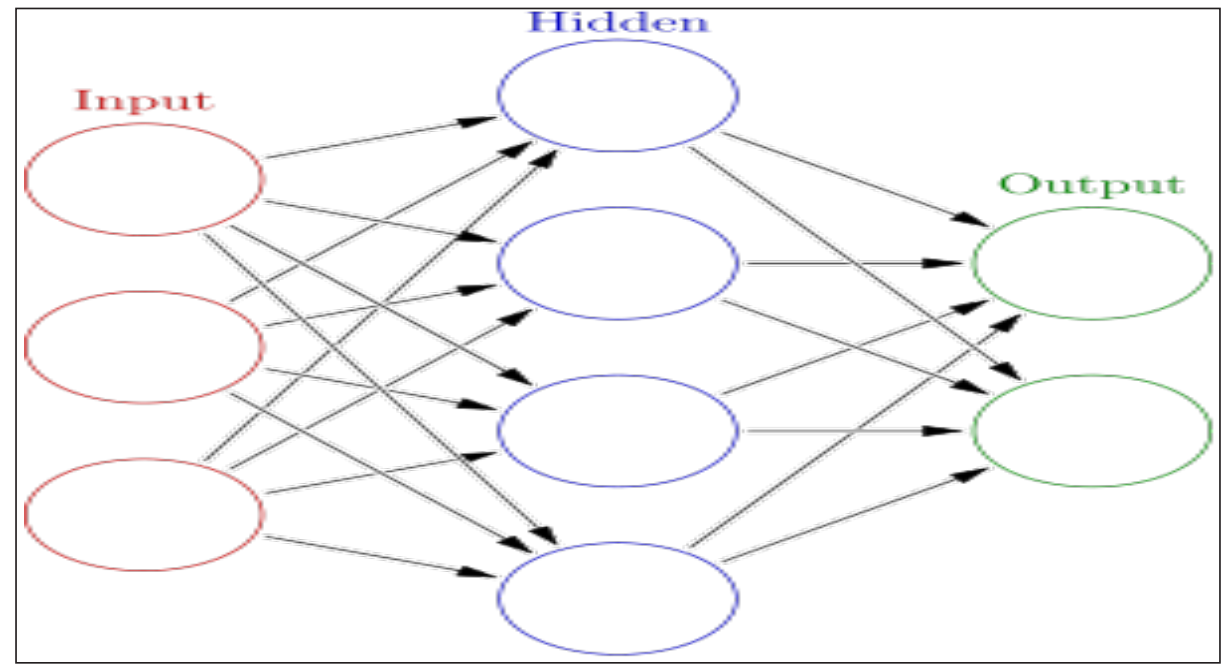

Fig. 5: Artificial Neural Network

\section{(C) Hardware and Software tools used for Machine learning}

As a dominant method of training for large scale commercial cloud-based artificial intelligence, central processing units (CPU) has now been replaced by graphic processing units (GPU) with enhancements made by artificial intelligence (AI). An estimate by OpenAI revealed that large deep learning projects from AlexNet (2012) to AlphaZero (2017) increased the hardware compute 300,000 times with a doubling time trendline of 3.4 months. The software tools containing a variety of machine learning algorithms include free and open-source software like Caffe, Deep Speed, Mahout, Mallet, Neural Lab, Octave, Orange, Peri Data Language, R, ROOT and, Shogun. Some proprietary software is also in high demand like Amazon Machine Learning, IBM Data Science Experience, Google Prediction API, IBM SPSS Modeler, Mathematica, MATLAB, Microsoft Azure, Neural Designer, Oracle Data Mining, and SAS Enterprise Miner to name a few.

\section{(D) Application of Machine Learning in Various Fields:}

Machine learning finds application in diverse fields like agriculture, anatomy, banking, bioinformatics, economics, financial market, insurance, marketing, online advertising, software engineering, search engine, 
telecommunication, and, optimization. It also finds application in some technology-intensive fields like adaptive websites, DNA Sequence, internet and credit card fraud detection, handwriting detection, robot locomotion, machine perception, and, structural health monitoring. In short, machine learning has been useful in almost all aspects of technology-driven modern life.

\section{APPLIED MACHINE LEARNING IN HEALTHCARE}

Machine Learning offers an objective opinion to our health problems. Earlier doctors used to prescribe solutions that were more subjective nature. They were regarded as Gods. Often wrong treatment led to unhappy incidents as well leading to maligning the doctors. But days have changed. Recent advancements in machine learning by Google's algorithms detected cancer tumour cells in the mammogram. [30] Stanford's deep learning algorithm detected skin cancer. Even diabetic retinopathy which is very common for diabetic patients has been successfully detected by deep learning of the retina images. Clinical decision-making has been more precise with an application of machine learning. If machine learning is reproducible and standardized and a proper algorithm can be developed, large image datasets in radiology, cardiology, and, pathology departments can be processed effectively and abnormalities can be detected very quickly and reliably. This will result in an efficient and effective health management system. Health information technology depends much on machine learning today. By using electronic medical records (EMR) and electronic health records (EHR) and feeding these records on machine learning platforms, doctors can deliver faster and reliable healthcare. As a corollary an integrated health delivery system can be developed and preventive screening of ailments can be suggested as well.

\section{(A) The Real-World Benefits of Machine in Healthcare}

Days are gone when medical records were mostly handwritten. Now every patient's medical records, diagnosis, treatment, prophylactic and therapeutic suggestions are all maintained electronically. Hence the artificial intelligence-based application of machine learning is the order of the day. In the real world, it will have a huge level of the successful application by the power of analytics. Through the advent and popularization of health information technology, machine learning can pave the way for more reliable, affordable, and, effective healthcare delivery [29]. Machine learning can do job unthinkable for any human doctor. Big data analytics and processing of patient diagnostic reports machine learning can provide precision treatment at the patient's doorsteps $24 / 7$ all over the world. This is the healthcare revolution of the $21^{\text {st }}$ century.

\section{(B) The Ethics of using Algorithms in Healthcare}

Ethics tells us what we should do and what not. Medical ethics in machine learning involves the decision by the doctor to prioritize which areas he needs to take help. Data about the patient's radiology reports can be assessed by the machine learning by it's the doctor who should analyze the patient's treatment options. Everything should not be kept in the hands of the machine as that can result in bias and wrongful treatment. Machine learning will help the doctors to understand better and faster. But the interpretation will be uniquely done by doctors as the case may differ from patient to patient even for the same disease and the same line of treatment [33]. Moreover, passion, care, and, human touch are important components 
of healthcare. Hence doctors should remember that and use machine learning judiciously as and when required for faster, more reliable, and, affordable healthcare that benefits every stakeholder.

\section{(C) Data Driven Machine Learning in Healthcare}

The Predictive algorithm is the bedrock for an effective machine learning process [22]. Hence, data should be reliable and accessible for proper medical intervention. Datasets should be large enough for proper results. Hence a concerted effort should be made for reliable collection of national and regional data and integrate with global data to come for an accurate prediction in case of common ailments. For rare diseases also the data integration is important. The Data collection process should also be transparent and trustworthy with no regional bias or manipulation. As machine learning is entirely data-driven, the accuracy and coverage of data are very important. Regional data hubs can be set up and third-party data centers can also be integrated to develop powerful algorithms. A Collaborative approach for a unified medical world that caters to mankind in a trustworthy manner is the benefit that can be reaped through machine learning [28].

\section{(D) Machine Learning from Theoretical to Clinical Reality:}

Theoretical machine learning can be translated into clinical reality if it's embedded [26]. Doctors treating patients in operation theatre in a Delhi hospital can get a real-time update on what's happening with a similar type of patient in Dallas or Durban. Hence this embedded technology will result in reliable healthcare certified across the medical fraternity. Not only the treatment will be world-class, the findings of this exercise will also enrich the health information technology for the benefit of subsequent patients worldwide. It sounds futuristic but machine learning technology and analytics can make this a reality very soon.

\section{(E) The Life Saving Technology that will Transform Healthcare}

The critical healthcare assessment system involves predictive, proactive, and preventive in that succession [25]. It will transform healthcare from reactive to proactive. There are unlimited opportunities for machine learning in healthcare as enlisted below:

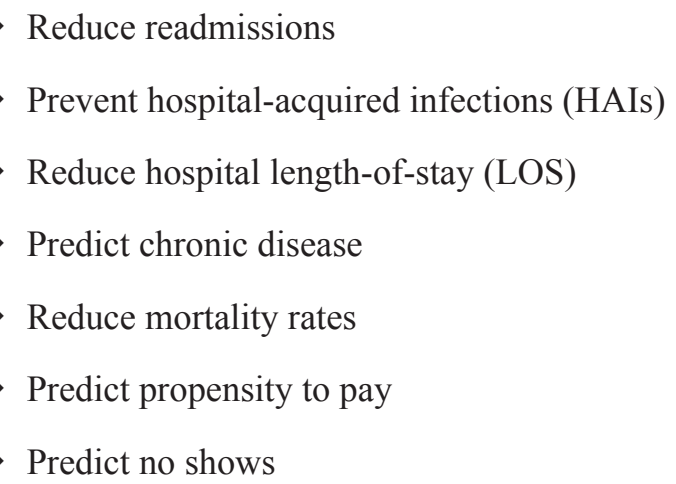

Reduce readmissions

Prevent hospital-acquired infections (HAIs)

Reduce hospital length-of-stay (LOS)

Predict chronic disease

Reduce mortality rates

Predict propensity to pay

Predict no shows 


\section{LIMITATIONS OF MACHINE LEARNING}

Machine learning has been transformative in some fields of research and practical applications but it has got some limitations as well [27]. The most noted limitation is that it failed to deliver the expected results. Lack of suitable data, lack of data access, data bias, privacy issues, improper choice of algorithms, inappropriate tools, and, evaluation problems led to ineffective machine learning [6]. For instance, in the year 2018, a self-driving car of famous car rental company Uber failed to recognize a pedestrian and killed him after a collision in the USA and similar kinds of incidents of machine learning failure had also been reported from Singapore and Taiwan. IBM Watson also failed to apply machine learning in healthcare even though years of research and billions of dollars have been invested behind it [20].

\section{SUGGESTIONS}

Deliberations in this paper suggest that machine learning has multiple benefits to modern healthcare management. Health information technology calls for intelligent systems that can predict the future of patients' vulnerability to chronic and crippling ailments. With the aid of machine learning technology, doctors can compute the diagnostic outcomes over digital platforms that can handle big data through the use of modern artificial intelligence and then correlate the same over cloud-based healthcare platforms. The results are multiple benefits in terms of disease management, choice of therapeutic and prophylactic measures in a more meaningful way. Medicos will still be in demand but the repetitive interventions that need intelligent systems to compute in the least time, effort, and the cost will increase the efficiency of medicos, and, hence the entire medical fraternity will be benefitted. Patients can receive state of the art diagnosis, predictive medications, and, suggestion in almost no time. Hence it is of paramount importance that the discipline of medicine takes the input of machine learning in a big way to solve today's pressing issues of healthcare management. This "benefits multiplier" of machine learning to healthcare is a formidable boon of modern technology to mankind.

\section{CONCLUSION}

It can be concluded that machine learning has a multiplier effect in modern healthcare. The benefits of machine learning in healthcare delivery are manifold. As the modern healthcare system depends much on diagnosis and patient's medical records, machine learning can be employed for processing of these large datasets and prescribe a meaningful report [17]. Doctors will always be in demand since they get a first-hand interaction with the patients. But their role will be less subjective and more objective [24]. This objectivity will increase the quality of medical treatment as well. With health information technology getting incorporated in every hospital and healthcare facilities across the world, the scientific, trustworthy, and, fast processing of this information to come to a reliable and meaningful conclusion can e effectively done by machine learning [13]. Advancement in artificial intelligence made machine learning see the light of the day. It's on us to understand this reliable technology and employ it for the betterment of healthcare delivery [18]. Plenty of reliable software tools are available in machine learning. An application of these tools and integrating these with the large medical datasets across the globe can transform the global healthcare system. The Economies of scale principle in Keynesian economics postulates the success of this global ramification of goods and services. Every stakeholder will be benefitted because precious resources like time and money will be saved. Precious lives will also be saved [19]. Efficiency and 
effectiveness will be achieved. With proper ethical practices and embedded technology days are very near when the patients, the world over will receive care as per global best practices on health. More research will bring more light in this area and advancements in other areas of science and technology accentuate this multiplier effect [21]. Health information technology coupled with machine learning and artificial intelligence is poised a transformative revolution in modern healthcare and it'll be a seamless activity for healthy, peaceful, and, progressive mankind.

\section{ACKNOWLEDGEMENTS}

Special thanks to Dr. P.K. Paul, Department of Computer and Information Science, Raiganj University, West Bengal, India - 733134 for his motivation and guidance.

\section{REFERENCES}

1. Afshin, R. and Talwalkar, A. 2012. Foundations of Machine Learning. USA, Massachusetts: MIT Press.

2. Agrawal, R., Imieliński, T. and Swami, A. 1993. "Mining association rules between sets of items in large databases". Proceedings of the 1993 ACM SIGMOD international conference on Management of Data - SIGMOD ‘93. p. 207.

3. Alex, R., Stephen, B. and Varma, P. 2019. "Weak Supervision: The New Programming Paradigm for Machine Learning". Hazy Research.

4. Alpaydin, E. 2010. Introduction to Machine Learning. MIT Press, pp. 9.

5. Bishop, C.M. 2006. Pattern Recognition and Machine Learning, Springer.

6. Bloomberg.com. 2016. "Why Machine Learning Models Often Fail to Learn: QuickTake Q\&A". Bloomberg.com. [2016-11-10].

7. Bozinovski, S. 1981. "Teaching space: A representation concept for adaptive pattern classification" COINS Technical Report No. 81-28, University of Massachusetts, MA.

8. Bozinovski, S. 1982. "A self-learning system using secondary reinforcement". Cybernetics and Systems Research: Proceedings of the Sixth European Meeting on Cybernetics and Systems Research. NorthHolland, pp. 397-402.

9. Bozinovski, S. 2001. "Self-learning agents: A connectionist theory of emotion based on crossbar value judgment." Cybernetics and Systems, 32(6): 637-667.

10. Bozinovski, S. 2014 "Modelling mechanisms of cognition-emotion interaction in artificial neural networks, since 1981," Procedia Computer Science p. 255-263.

11. Brazdil, P., Carrier, C.G., Soares, C. and Vilalta, R. 2009. Meta-learning: Applications to Data Mining ( $4^{\text {th }}$ ed.). Springer Science Business Media, pp. 10-14.

12. Bzdok, D., Altman, N. and Krzywinski, M. 2018. "Statistics versus Machine Learning". Nature Methods, 15(4): 233-234. 
13. Chandola, V., Banerjee, A. and Kumar, V. 2009. “Anomaly detection: A survey". ACM Computing Surveys, 41(3): 1-58.

14. Coates, A., Lee, H. and Andrew, Y. 2011. An analysis of single-layer networks in unsupervised feature learning. Int'l Conf. on AI and Statistics (AISTATS).

15. Dokas, P., Ertuz, L., Kumar, V., Lazarevic, A., Srivastava, J. and Tan, P. 2002. “Data mining for network intrusion detection". Proceedings NSF Workshop on Next Generation Data Mining.

16. Duda, R. and Hart, P. 1973. Pattern Recognition and Scene Analysis, Wiley Interscience.

17. Friedman, J.H. 1998. "Data Mining and Statistics: What's the connection?". Computing Science and Statistics, 29(1): 3-9.

18. Gareth, J., Daniela, W., Trevor, H. and Robert, T. 2013. An Introduction to Statistical Learning, Springer, pp. vii.

19. Goldberg, D.E. and Holland, J.H. 1988. "Genetic algorithms and machine learning”. Machine Learning, 3(2): 95-99.

20. Hernandez, D. and Greenwald, T. 2018. “IBM Has a Watson Dilemma”. Wall Street Journal, pp. 08-11.

21. Jordan, M.I. 2014. "Statistics and machine learning”, Reddit.

22. Jordan, M.I. and Bishop, C.M. 2004. "Neural Networks". Computer Science Handbook, Second Edition (Section VII: Intelligent Systems). Boca Raton, Florida: Chapman \& Hall/CRC Press LLC.

23. Kohavi, R. and Provost, F. 1998. "Glossary of terms," Machine Learning, 30(2-3): 271-274.

24. Langley, P. 2011. “The changing science of machine learning”. Machine Learning, 82(3): 275-279.

25. Michie, D., Spiegelhalter, D.J. and Taylor, C.C. 1994. "Machine Learning, Neural, and, Statistical Classification". Ellis Horwood Series in Artificial Intelligence.

26. Mitchell, T. 1997. Machine Learning. McGraw Hill, pp. 2.

27. Nicolas, L.R., Bengio, Y. and Fitzgibbon, Andrew. 2012. "Improving First and Second-Order Methods by Modelling Uncertainty”. Optimization for Machine Learning. MIT Press, pp. 404.

28. Nilsson, N. 1965. Learning Machines, McGraw Hill.

29. Otterlo, M. and Wiering, M. 2012. Reinforcement learning and Markov decision processes. Reinforcement Learning, Adaptation, Learning, and Optimization, 12: 3-42.

30. Samuel, A. 1959. "Some Studies in Machine Learning Using the Game of Checkers". IBM Journal of Research and Development, 3(3): 210-229.

31. Sarle, W. 1994. "Neural Networks and statistical models". CiteSeerX 10.1.1.27.699.

32. Stuart, R. and Peter, N. 2003. Artificial Intelligence: A Modern Approach (2 ${ }^{\text {nd }}$ ed.). Prentice-Hall.

33. Vinod. K. 2012. “Do We Need Doctors or Algorithms?”. Tech Crunch. [January 10, 2012].

34. Bengio, Y. 2009. Learning Deep Architectures for AI. Now Publishers Inc., pp. 1-3. 